\title{
Aptamers (Chemical Antibodies) for the Mitigationof Mycotoxins in Domestic Livestock
}

\author{
Srinivasa Madhyastha* and Ronald R Marquardt \\ 71 Loyola Bay, Winnipeg, Manitoba, Canada
}

Submission: August 14, 2018; Published: August 23, 2018

*Corresponding author: Srinivasa Madhyastha, 71 Loyola Bay, Winnipeg, Manitoba, Canada R3T 3J7, Canada, Tel: +204-269-1483;

Email: srim@shaw.ca

\begin{abstract}
Aptamers are short, single-stranded DNA or RNA molecules (oligonucleotides) that bind to target molecules, including toxins with high affinity and specificity. For the first time, aflatoxin B1(AFB1)-specific DNA aptamer was used as a toxin binder (adsorbent) to evaluate its ability to neutralize the toxicity in zebrafish embryos treated with AFB1. While AFB1 was highly toxic to Zebrafish embryos, aptamer alone was not toxic. However, AFB1 when bound by the aptamer was completely non-toxic when given to Zebrafish embryos. Thus, aptamers have the potential to protect domestic livestock against AF1 toxicity and from other mycotoxins.
\end{abstract}

Keywords: Aptamer; Oligonucleotide; Mycotoxin; Aflatoxin B1; Toxicity neutralization; Zebrafish; Livestock feed; Penicillium; Aspergillus; Fusarium; Oligonucleotides; Peptides; Deoxynivalenol; Trichothecenes; Zearalenone; Mortality

\section{Introduction}

Mycotoxins are secondary metabolites of fungi, which are toxic to animals at certain concentrations. The primary classes of mycotoxins are aflatoxins (AFBs), zearalenone, trichothecenes (including deoxynivalenol), fumonisins, and ochratoxin A (OTA) [1]. The major mycotoxin-producing fugal genera are Aspergillus, Fusarium and Penicillium. Certain fungi can grow and produce mycotoxins in many growing crops while other mycotoxins are produced during improper storage of grains. The Food and Agriculture Organization (FAO) has estimated that over $25 \%$ of the world's crops are affected by mycotoxins each year [2]. Annual losses in the US and Canada due to the impact of mycotoxins on the livestock and feed industries are estimated at $\$ 5$ billion USD [3]. Mycotoxins exhibit a variety of biological effects in livestock, which include liver and kidney toxicity, and neurological, estrogenic and teratogenic effects. Some mycotoxins such as AFB1, OTA and FB1 are carcinogenic. Additionally, the consumption of mycotoxin-contaminated feed by livestock can cause loss of appetite, decreased feed efficiency, feed refusal, poor weight gain, immunosuppression, and mortality [4]. In general, consumption of mycotoxins results in reduced animal performance including decreased production of flesh or eggs in poultry, milk in dairy cows and meat in pigs and beef cattle.

The transfer of mycotoxins such as the AFBs and OTA into edible animal products (milk, meat, offal, eggs) can have detrimental effects on human health. Currently, mycotoxin binders (adsorbents) are widely used to detoxify mycotoxins in domestic livestock feed. These binders are non-specific, partially detoxify only certain mycotoxins and adsorb important nutrients, such as vitamins, minerals and antioxidants in feed. The objective of the current study was to determine if specific AFB1 binding aptamers can neutralize the toxicity of AFB1 to prevent the lethality in Zebrafish embryos. Aptamers (chemical antibodies) are short, single-stranded DNA or RNA oligonucleotides or peptides that have been engineered through a selection process to exhibit high binding affinity and specificity to their target [5]. Aptamers withstand a wide range of temperatures and they can be modified during the selection process for persistence under low or high $\mathrm{pH}$ and for preventing the degradation by nucleases. Aptamers are currently used for the determination of mycotoxins as they can be designed to specifically bind a wide range of mycotoxins with high affinity [6]. Furthermore, the authors are not aware of any research reports to date demonstrating the ability of aptamers to neutralize the toxic effects of mycotoxins using an animal model.

\section{Materials and Methods}

Aflatoxin B1 was obtained from Cayman Chemical Company, Ann Arbor, MI, USA. AFB1-specific DNA aptamer was from NeoVentures Biotechnology Inc., London, ON, Canada. AFB1 lethality studies with Zebrafish embryos were carried out by Phylonix Pharmaceutical Inc., Cambridge, MA, USA. Zebrafish embryos were generated by using a Mass Embryo Production System. Embryos were cleaned by removing dead embryos and sorted by developmental stage. As embryos receive nourishment from an attached yolk sac, no feeding was required for 6-days 
post fertilization (dpf). Aflatoxin B1 stock solution was prepared in DMSO and added to the fish water as shown in Table 1 at a final concentration of $0.2 \mu \mathrm{M}$ AFB1 and $0.1 \%$ DMSO. Aflatoxin B1 specific aptamer Master Stock (MS) solution was prepared by dissolving a known quantity ( 350 nmoles $)$ of aptamer powder in $2.8 \mathrm{ml}$ fish water to generate a $125 \mu \mathrm{M}$ aptamer MS. $40 \mu \mathrm{l}$ of $125 \mu \mathrm{M}$ aptamer MS solution was pre-incubated with $4 \mu \mathrm{l}$ of $0.25 \mathrm{mM}$ AFB1 in 6-well microplates containing $1956 \mu \mathrm{l}$ fish water/well for $15 \mathrm{~min}$. After pre-incubation, $2 \mathrm{ml}$ fish water containing $2 \mathrm{dpf}$ self-hatched zebrafish $(\mathrm{N}=30)$ was added to the pre-incubated solution to yield a final volume of $4 \mathrm{ml}$. The final concentration of aptamer was $1.25 \mu \mathrm{M}, 5$-fold higher than final AFB1 concentration. Two dpf zebrafish were exposed continuously to each condition for $96 \mathrm{hr}$. The final treatment concentrations in $4 \mathrm{ml}$ fish water are shown in Table 1 . The final DMSO concentration was $0.1 \%$ for all treatment conditions. The experiment was repeated three times.

Table 1: Final aflatoxin B1 (AFB1) and aptamer treatment concentrations in the Zebrafish embryo lethality test1.

\begin{tabular}{|c|c|c|c|}
\hline S. No & $\begin{array}{c}\text { Treatment No. } \\
\boldsymbol{\&} \text { Reagents }\end{array}$ & $\begin{array}{c}\text { Final Conc. of } \\
\text { AFB1 }(\boldsymbol{\mu} \mathbf{M})\end{array}$ & $\begin{array}{c}\text { Final conc. of } \\
\text { AFB1-Specific } \\
\text { Aptamer }(\boldsymbol{\mu} \mathbf{M})\end{array}$ \\
\hline 1 & $0.1 \%$ DMSO & 0 & 0 \\
\hline 2 & $\begin{array}{c}\text { AFB1- Specific } \\
\text { Aptamer + } \\
0.1 \% \text { DMSO }\end{array}$ & 0 & 1.25 \\
\hline 3 & $\begin{array}{c}\text { AFB1+0.1\% } \\
\text { DMSO }\end{array}$ & 0.25 & 0 \\
\hline & $\begin{array}{c}\text { AF- } \\
\text { B1+AFB1-Spe- } \\
\text { cific Aptamer + } \\
0.1 \% \text { DMSO }\end{array}$ & 0.25 & 1.25 \\
\hline
\end{tabular}

${ }^{1}$ Dead Zebrafish were counted daily and removed. After treatment for $96 \mathrm{hr}$, total lethality was calculated. To obtain mean and Standard Deviation (SD) for each condition, experiments were performed three times.

Table 2: Lethality of Zebrafish embryos administered different combinations of aflatoxin B1 (AFB1) and aptamer1.

\begin{tabular}{|c|c|c|c|c|c|}
\hline \multicolumn{7}{|c|}{ Percent Lethality } \\
\hline $\begin{array}{c}\text { Final Treat- } \\
\text { ment Concen- } \\
\text { tration }(\mu \mathrm{\mu M})\end{array}$ & Exp. 1 & Exp. 2 & Exp. 3 & Mean & SD \\
\hline $0.1 \%$ DMSO & $0(0 / 30)$ & $0(0 / 30)$ & $0(0 / 30)$ & 0 & 0 \\
\hline $\begin{array}{c}\text { AFB1-Aptamer + } \\
0.1 \% \text { DMSO }\end{array}$ & $0(0 / 30)$ & $0(0 / 30)$ & $0(0 / 30)$ & 0 & 0 \\
\hline $\begin{array}{c}\text { AFB1 + 0.1\% } \\
\text { DMSO }\end{array}$ & $\begin{array}{c}80 \\
(24 / 30)\end{array}$ & $\begin{array}{c}83.3 \\
(25 / 30)\end{array}$ & $\begin{array}{c}83.3 \\
(25 / 30)\end{array}$ & 82.2 & 1.9 \\
\hline $\begin{array}{c}\text { AFB1 + } \\
\text { AFB1-Specific } \\
\text { Aptamer + 0.1\% } \\
\text { DMSO }\end{array}$ & $0(0 / 30)$ & $0(0 / 30)$ & $0(0 / 30)$ & 0 & 0 \\
\hline
\end{tabular}

${ }^{1}$ Numbers in Parentheses: Number of dead Zebrafish embryos divided by number of Zebrafish embryos per well.

\section{Results and Discussion}

After treatment for 96hrs, zero percent lethality was observed in Zebrafish embryos when treated with $0.1 \%$ DMSO alone, validating the assay. Zero percent lethality was also observed after treatment with AFB1-specific aptamer alone $+0.1 \%$ DMSO confirming that AFB1-specific aptamer did not induce lethality in zebrafish. However, $82.2 \pm 1.9 \%$ lethality was observed in Zebrafish embryos after treatment with AFB1 alone $+0.1 \%$ DMSO. In contrast, zero percent lethality was observed after treatment with the AFB1 and AFB1-specific aptamer combination (AFB1+AFB1-Specific Aptamer + 0.1\% DMSO), indicating that AFB1-specific aptamer neutralized 100\% AFB1 toxicity induced lethality in Zebrafish embryos (Table 2). These data demonstrate that aptamers designed to specifically bind AFB1 are capable of completely neutralizing the effects of AFB1 in a Zebrafish embryo model. Thus, as is evident from this study, specific aptamers are highly effective in neutralizing the toxic effects of AFB1 and they should be capable of detoxifying other mycotoxins in animals that are fed diets containing mycotoxins. Aptamers may be degraded by nucleases produced by intestinal microorganisms. This problem can be overcome using mirror image DNA or RNA aptamers, called Spiegelmers ${ }^{\circledR}$, that are resistant to all nucleases [7]. Spiegelmer ${ }^{\circledR}$ aptamers can be produced at a relatively low cost in large quantities. A comprehensive comparison of the advantages and disadvantages of nucleic acid aptamers versus antibodies has been reviewed by Zhou and Rossi [5]. Spiegelmers ${ }^{\circledR}$ (mirror image DNA or RNA aptamers) have the potential to specifically and safely neutralize the toxicity of mycotoxins present in livestock feeds.

\section{Conclusion}

AFB1 specific DNA aptamer was able to bind AFB1 and eliminate AFB1 toxicity in Zebrafish embryos. Thus, other aptamers that are specific to selected mycotoxins should also be able to neutralize the toxic effects of mycotoxins in a Zebrafish embryo model and in domestic livestock. A considerable amount of research must be carried out to develop cost effective commercial means of using aptamers to mitigate the effects of mycotoxins present in livestock feeds. Aptamer-based mycotoxin binders have the potential to meet the unmet mycotoxin detoxifiers market need for a safe, specific and cost-effective treatment capable of neutralizing $100 \%$ of mycotoxin toxicity. Thus, aptamer-based binders have a promising future as they could be widely used to bind and neutralize not only AFB1 but also other mycotoxins in the feed of domestic livestock.

\section{References}

1. Chaytor AC, Hansen JA, Heugten EV, Todd SM, Kim SW (2011) Occurrence and decontamination of mycotoxins in swine feed. AsianAustralasian Journal of Animal Sciences 24(5): 723-738.

2. Richard JL, Payne GA (2003) Council for Agricultural Science and Technology Task (CAST) Force Report No. 139, Mycotoxins: Risks in Plant Animal and Human Systems, Ames, IA, USA. 
3. Charmley LL, Trenholm HL, Prelusky DB (1995) Mycotoxins: their origin, impact and importance: insights into common methods of control and elimination. In: Biotechnology in the Feed Industry, Proceedings of Alltech's Eleventh Annual Symposium. Lyons TP, Jacques KA (Eds.), p. 41-63.

4. Zaki MM, El-Midany SA, Shaheen HM, Rizzi L (2012) Mycotoxins in animals: Occurrence, effects, prevention and management. Journal of Toxicology and Environmental Health Sciences 4: 13-28.
5. Zhou J, Rossi J (2017) Aptamer as targeted therapeutics: current and potential challenges. Nature Reviews Drug Discovery 16(3): 181-202.

6. Urusov AE, Zherdev AA, Petrokova AV, Sadykhov EG, Koroleva OV, et al. (2015) Rapid multiple immune enzyme assay of mycotoxins. Toxins 7 238-254.

7. Vater A, Klassmann S (2015) Turning mirror-image oligonucleotides into drugs: the evaluation of Spiegelmer ${ }^{\circledR}$ therapeutics. Drug Discovery Today 20(1): 147-155.
This work is licensed under Creative Commons Attribution 4.0 License DOI: 10.19080/JDVS.2018.07.555714 